\title{
Rotating a Curvaton Brane in a Warped Throat
}

\author{
Jun Zhang ${ }^{a *}$, Yi-Fu Cai ${ }^{b \dagger}$, and Yun-Song Piao ${ }^{a \ddagger}$ \\ ${ }^{a}$ College of Physical Sciences, Graduate University of Chinese Academy of Sciences, Beijing 100049, China and \\ ${ }^{b}$ Institute of High Energy Physics, Chinese Academy of Sciences, P.O.Box 918-4, Beijing 100049, P.R.China
}

\begin{abstract}
In this paper we study a curvaton model obtained by considering a probe anti-D3-brane with angular motion at the bottom of a KS throat with approximate isometries. We calculate the spectrum of curvature perturbations and the non-Gaussianities of this model. Specifically, we consider the limit of relativistic rotation of the curvaton brane which leads to a small sound speed, and thus it can be viewed as an implementation of the DBI-curvaton mechanism. We find that the primordial power spectrum is nearly scale-invariant while the non-Gaussianity of local type is sizable and that of equilateral type is usually large and negative. Moreover, we study both the theoretical and observational constraints on this model, and find that there exists a sizable allowed region for the phase space of this model.
\end{abstract}

\section{INTRODUCTION}

The curvaton mechanism [1] is an interesting proposal for explaining the observed scale-invariant primordial density perturbations in the framework of inflation (and some pioneering works on this mechanism have been considered in Refs. 2, 3, 4] ). In this scenario, at least two fields are required, in which the curvaton field is subdominant during inflation but becomes dominant after the inflaton decays. When the curvaton is subdominant it provides entropy (iso-curvature) perturbations during inflation, and afterwards, these entropy perturbations can be converted to curvature perturbations when the curvaton starts to dominate the universe. After the curvaton decays, the universe enters the standard thermal history, and then the primordial curvature perturbations lead to the formation of the large scale structure of our universe [5, 6].

Recently, a curvaton scenario realized in the frame of stringy inflationary models was presented in Ref. 7]. As advocated in Ref. [8], a system of D- and anti-D-branes in a warped throat can be made use of to provide an inflationary stage in the early universe [9], and its dynamics is described by a Dirac-Born-Infeld (DBI) action in the relativistic limit [10, 11]. Therefore, the curvaton mechanism realized in this background is the so-called "DBIcurvaton", where the curvaton field can be interpreted as a moduli parameter of a D-brane moving in a warped throat 12]. However, it is widely recognized that a brane inflation model of DBI-type often suffers from problems of backreaction both from the relativistic brane moving in the warped throat 10, 13, 14] and from the inflationary background (see [15] for an extended discussion), and so is often unable to provide a enough long inflationary stage required by cosmological observations [16, 17, 18]. The original DBI-curvaton model also suffers from the same problem as that of DBI inflation.

\footnotetext{
*Email: zhangjun408@mails.gucas.ac.cn

${ }^{\dagger}$ Email: caiyf@ihep.ac.cn

$\ddagger$ Email: yspiao@gucas.ac.cn
}

To circumvent this problem, one has to finely tune the precise shape of the potential of the inflaton in the presence of supersymmetrically embedded D7-branes and an anti D3- brane localized at the tip of the warped conifold cone, and therefore the resulting model is rather delicate as studied in Refs. [19, 20, 21]. Another approach to easing this tension suggests that the motion of a probe brane along the angular coordinates in the internal space can be taken into account to prolong the inflationary period 22] which relaxed the theoretical constraints from the gravitational backreaction, and so extends the phase space of brane inflation [14].

In this paper, we study a new DBI-type curvaton model established on the scenario of multiple brane inflation proposed in Refs. 23, 24]. We suggest that a probe anti-D3-brane with angular momentum on the tip of an approximately isometric warped throat can play the role of a curvaton field. Specifically, we consider the well-known Klebanov-Strassler (KS) throat 25] which is a nonsingular deformed conifold with its moduli being stabilized by fluxes [26, 27]. In this model, the anti-D3brane always sits at the bottom of the deformed throat due to the sum of gravitational and gauge forces. Correspondingly its radial position is nearly fixed and the angular motion can be safely preserved during inflation.

Since the specific realization of this curvaton mechanism involves at least two branes with the anti-D3-brane as the curvaton brane, this model can be viewed as an application of the multi-brane inflation model 23, 24, 28], and it naturally possesses some features of the multibrane inflation model. For instance, the spectral index of iso-curvature perturbations generated by the curvaton brane during inflation has a slight red tilt deviating from scale-invariance, and is mainly dependent on the slowroll parameter characterizing the variation of the Hubble parameter during inflation. At cubic order we find the nonlinear perturbations of a curvaton brane model have the combined features of a usual curvaton model and a DBI-inflation one. The non-Gaussianity of local type nicely agrees with the traditional result obtained in a general curvaton model; however, the non-Gaussianity of equilateral type is amplified by the small sound speed of the curvaton brane which is consistent with the usual 
scenario of brane inflation.

Our paper is organized as follows. In Section II we briefly review the dynamics of D- and anti-D-branes in the KS throat. In Section III, we present the realization of the DBI-curvaton in the KS throat and show that the curvaton brane can rotate in the relativistic limit. In Section IV we calculate the curvature perturbations and the non-Guassianities generated in this model. The consistency relationships are analyzed in Section V. Section VI contains discussion and conclusions.

\section{BRANE DYNAMICS IN A WARPED THROAT}

To begin with, we consider a flux compactification of type IIB string theory on an orientifold of a Calabi-Yau three-fold[26]. The geometry of this warped deformed conifold is viewed as a solution to type IIB supergravity with the presence of negative tension sources. It can be explicitly constructed by taking a stack of D3-branes with $M$ units of the Ramond-Ramond (RR) fluxes $F_{3}$ on the $S_{3}$ (A-cycle) and $K$ units of the Neveu-Schwarz-NeveuSchwarz (NS-NS) fluxes $\mathrm{H}_{3}$ on the dual cycle (B-cycle) which is the $S_{2}$ times a circle extended along the radial direction. This gives,

$$
\frac{1}{2 \pi \alpha^{\prime}} \int_{A} F_{3}=2 \pi M, \quad \frac{1}{2 \pi \alpha^{\prime}} \int_{B} H_{3}=-2 \pi K,
$$

where $\alpha^{\prime}$ is related to the string mass scale by $\alpha^{\prime}=1 / m_{s}^{2}$. When the fluxes are turned on, they warp the geometry of the conifold and the background metric of the 10D spacetime can be expressed as follows,

$$
d s^{2}=h^{2} g_{\mu \nu} d x^{\mu} d x^{\nu}+h^{-2}\left(d l^{2}+l^{2} \tilde{g}_{m n} d y^{m} d y^{n}\right),
$$

where the warping factor $h$ can be a function of the radius $l$ and internal coordinates $y^{m}$ generically. Additionally, $g_{\mu \nu}$ is the metric of 4-dimensional spacetime and $\tilde{g}_{m n}$ characterizes the internal space of the compact manifold.

In the above system, we have only introduced the D3branes. The geometry away from the tip of the conifold is an approximately Anti-de-Sitter (AdS) form with

$$
h \simeq \frac{l}{L},
$$

and the characteristic length scale $L$ is fixed by the flux number as

$$
L^{4} \simeq \frac{27}{4} \pi g_{s} M K \alpha^{2},
$$

where $g_{s}$ is the string coupling which has to satisfy $g_{s} M \gg 1$ to ensure the validity of the supergravity description 29]. In order to obtain a de-Sitter (dS) solution which may be used to realize inflation, we can introduce anti-D3-branes at the tip of the throat, which can lift the AdS vacuum such as the KKLT mechanism[27].
Note that, the warping factor at the infrared (IR) end of the throat takes

$$
h_{I R} \simeq \exp \left(-\frac{2 \pi K}{3 g_{s} M}\right),
$$

and the IR cutoff of the radial coordinate $l_{I R} \simeq$ $\left(g_{s} M \alpha^{\prime}\right)^{1 / 2} h_{I R}$.

Now, we are interested in the dynamics of the branes in the KS throat. It has been shown that the $\overline{D 3}$-brane will sink to the tip of the throat, while the D3-brane is almost free except attracted by the $\overline{D 3}$-brane only. To have a quick look, we can write down the effective action for a D3-brane or an $\overline{D 3}$-brane [30],

$$
S=-T_{3} \int d^{4} x \sqrt{-\operatorname{det}\left[G_{\mu \nu}\right]} \pm T_{3} \int d^{4} x\left(C_{4}\right)_{0123},
$$

with $G_{\mu \nu}$ being defined as

$$
G_{\mu \nu}=G_{A B} \frac{\partial X^{A}}{\partial x^{\mu}} \frac{\partial X^{B}}{\partial x^{\nu}},
$$

where + is for D3-brane, - for $\overline{D 3}$, and the $T_{3}$ is the tension which is given by $T_{3}=\frac{m_{s}^{4}}{(2 \pi)^{3}} g_{s}$. The equation of motion of the RR field yields,

$$
\left(C_{4}\right)_{0123}=\sqrt{-g} h^{4} .
$$

So, if the transverse fluctuations of the brane vanish, we will find that, for D3, these two contributions to the action cancel exactly; however, they could sink $\overline{D 3}$ to the bottom of the throat.

As usual, if the KS throat is isometric, the warp factor $h$ is independent of the angular coordinates $y^{m}$. However, in a general case, there are some corrections which make the warp factor dependent on angular coordinates. For example, since the compact C-Y manifold cannot have exact continuous isometries, the isometries of the bulk must be broken when the finite throat is glued on this bulk, and then the warp factor becomes angulardependent. Since these effects are suppressed by powers of the warp factor, the correction to the warp factor can be described in terms of $\Delta\left(h^{4}\right) \sim h_{I R}^{\beta} f(y)$ as analyzed in Refs. [31, 32, 33]. However, we shall keep in mind that, $\Delta\left(h^{4}\right)$ can not be too large, otherwise the geometry of the KS throat would be distorted seriously.

A nonperturbative effect which stabilizes the Kähler moduli could bring a potential for the branes [34, 35]. The form of this potential depends on the precise embedding of the wrapped branes, and for embeddings which do not admit supersymmetric vacua on the tip, this potential could be angular-dependent. In Ref. [22], the authors have provided an estimate of the nonperturbative effect which is dominated by the warp factor in the case of the Kuperstein embedding [36] of the D7-brane, where the angular degrees of freedom obtain a mass term,

$$
m_{n p}^{2} \simeq \frac{h_{I R}^{2}}{g_{s} M \alpha^{\prime}} \frac{\epsilon}{\mu}
$$


in which $\mu$ measures the minimal radial location reached by the D7-brane, and $\epsilon \sim \mu h_{I R}^{3 / 2}$ characterizes the deformation of the coinfold.

To combine the corrections mentioned above, we can obtain the effective action of the $\bar{D} 3$-brane as follows,

$$
S=-T_{3} \int d^{4} x \sqrt{-\operatorname{det}\left[G_{\mu \nu}\right]}-T_{3} \int d^{4} x \sqrt{-g}\left(h^{4}+V_{n p}\right),
$$

where $V_{n p}$ stands for the angular-dependent potential given by the nonperturbative effects, and so is a function of the angular coordinates $y^{m}$. Note that we have neglected the interactions among the curvaton and other fields for simplicity. In addition, even if the isometries of the throat are broken, the dynamics of the brane along the radial direction are almost the same as that in the isometric throat, unless the bulk effects can distort the throat geometry drastically.

At the end of this section we would like to comment on the tadpole condition which is a limit on the net brane charge. Since the branes carry charges, they cannot be inserted into a compact space optionally. The extra brane charges must be compensated by the background, and thus the following condition has to be satisfied

$$
\frac{\chi}{24}=N_{D 3}-N_{\overline{D 3}}+\frac{1}{\kappa_{10}^{2} T_{3}} \int_{C-Y} H_{3} \wedge F_{3},
$$

where $\chi$ is the Euler number of the $\mathrm{C}-\mathrm{Y}$ manifold, and $N_{D 3}\left(N_{\overline{D 3}}\right)$ stands for the number of the D3-brane $(\overline{D 3}-$ brane). In the usual case, $\chi$ is of order $O\left(10^{5}\right)$ [37], and so it puts an upper bound on the background charge number as $M K \lesssim 10^{5}$.

\section{THE MODEL OF CURVATON BRANE}

Within the background introduced in the previous section, now we are able to construct the model of a DBIcurvaton from a $\overline{D 3}$-brane rotating in the KS throat. We consider a $\bar{D}$-brane with angular momentum at the tip of the KS throat which is approximately isometric. As shown in the previous section, this brane is localized in the radial direction. Note that in Ref. [14] it was found that the angular momenta of the branes could be inflated away if these branes were used to drive inflation. In our construction, we assume that the inflationary background is still driven by a D3-D7 system as introduced in Refs. 19, 21] (see [38, 39] for earlier studies), the radial coordinate of the $\bar{D}$-brane is nearly fixed, and there exists an external potential from the nonperturbative effect of moduli stabilization which drives the curvaton brane to rotate. Consequently, provided the external potential persists sufficiently long, the angular momentum of the probe brane can safely survive during inflation. ${ }^{1}$ In this

\footnotetext{
1 This is different from Ref. [14] in which their driving force is still almost radial and so leads to damping angular momenta. The
}

case, the radial coordinate of the probe brane is around $l_{I R}$, and thus we can study its dynamics along the angular directions at the tip of the KS throat and its application in the curvaton mechanism. The global scenario of our model is sketched in Fig. 1.

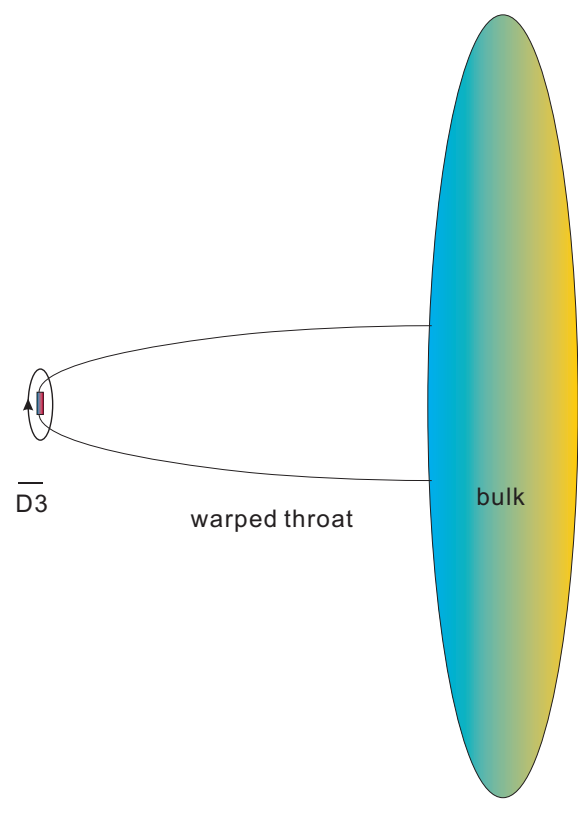

FIG. 1: A rotating $\overline{D 3}$-brane in a warped throat. In a deformed KS throat, the branes are no longer pointlike in the extra dimensions. The circle stands for the angular motion of the brane at the infrared tip of the warped throat, $l_{I R}$.

Substituting the induced metric on the $\bar{D} 3$-brane into its effective action (10), we can get a simplified form,

$$
\begin{aligned}
S \simeq & T_{3} \int d^{4} x \sqrt{-g}\left[-h^{4} \sqrt{1+\frac{1}{h^{4}}\left(l_{I R}^{2} \tilde{g}_{m n} \partial_{\mu} y^{m} \partial^{\mu} y^{n}\right)}\right. \\
& \left.-\left(h^{4}+V_{n p}\right)\right],
\end{aligned}
$$

where we have neglected the higher derivative terms on spatial directions which are secondary on affecting the background evolution and the primordial perturbations at large scales. Note that, although the terms including higher spatial derivatives are negligible at linear and even cubic order, they could affect the nonlinear evolution of primordial perturbations at fourth order and so could leave a significant imprint on the trispectra of this model. This will be studied in following works in the near future.

Moreover, there are three angular directions of the $S_{3}$ tip, and everyone is able to play the role of a curvaton field. We would like to pick up the flattest direction, and define it as the $y$ coordinate, for simplicity. Consequently, we can define the curvaton field as,

$$
\sigma \equiv l_{I R} \sqrt{T_{3}} y .
$$

non-vanishing angular degree of freedom in a warped throat was earlier applied to realize a bouncing universe [40, 41]. 
Finally, the effective action of the curvaton brane can be described by,

$$
\begin{aligned}
S \simeq & T_{3} \int d^{4} x \sqrt{-g}\left\{-h^{4}(\sigma) \sqrt{1+\frac{1}{h^{4} T_{3}}\left(\partial_{\mu} \sigma \partial^{\mu} \sigma\right)}\right. \\
& \left.-\left[h^{4}(\sigma)+V_{n p}(\sigma)\right]\right\} .
\end{aligned}
$$

As usual, we define a sound speed parameter of the curvaton field

$$
c_{s} \equiv \sqrt{1-\frac{2}{h^{4} T_{3}} X}
$$

where $X$ is define as $X \equiv-\frac{1}{2}\left(\partial_{\mu} \sigma \partial^{\mu} \sigma\right)$. Further, we would like to define an effective potential of the curvaton field as follows,

$$
V(\sigma) \equiv T_{3}\left(2 h^{4}+V_{n p}\right)
$$

and thus the corresponding effective Lagrangian can be written as,

$$
P \equiv T_{3} h^{4}\left(1-c_{s}\right)-V(\sigma)
$$

of which the form is similar to that of the usual DBI model.

We notice that a similar scenario involving a slowrotating $\overline{D 3}$-brane was considered in Ref. [42] and its action was expanded to quadratic order under the assumption of the non-relativistic limit $c_{s} \sim 1$. In the current work, however, we are going to consider the relativistic dynamics of the model of the curvaton brane with the limit of $c_{s} \sim 0$. In a flat Friedmann-RobertsonWalker (FRW) universe with the metric characterized by,

$$
d s_{F R W}^{2}=-d t^{2}+a^{2}(t) \delta_{i j} d x^{i} d x^{j},
$$

the equation of motion for the curvaton field $\sigma$ is given by

$$
\ddot{\sigma}+3 H \dot{\sigma}-\frac{\dot{c_{s}}}{c_{s}} \dot{\sigma}-c_{s} P_{, \sigma}=0
$$

where the dot represents the cosmic time derivative, and $P_{, \sigma}$ denotes the derivative of $P$ with respect to the scalar field $\sigma$. One may notice that, since the energy density of the curvaton is secondary during inflation, the hubble parameter $H$ is determined by the background D3-D7 inflationary model.

Before studying the cosmological perturbations of this model, we would like to study the evolution of its background equation under the relativistic limit. In the case of $c_{s} \sim 0$, Eq. (15) yields

$$
\dot{\sigma} \simeq-h^{2} \sqrt{T_{3}}
$$

and it implies that the variation of the curvaton is roughly $\Delta \sigma \sim-h^{2} \sqrt{T_{3}} \Delta t$ for a fixed time interval $\Delta t$. Besides, as mentioned in Section 2, provided the geometry of the KS throat is not distorted too much, we still have the infrared warp factor $h_{I R}$. Following Refs. [23, 24], we have an ansatz with the following form,

$$
\sigma=-h_{I R}^{2} \sqrt{T_{3}} t\left(1+\frac{\alpha}{(-t)^{p}}+\cdots\right)
$$

where we have set $t \rightarrow-\infty$ at the beginning of inflation. Moreover, Eq. (19) can be rewritten as

$$
\frac{d}{d t}\left(\frac{\dot{\sigma}}{c_{s}}\right)+3 H \frac{\dot{\sigma}}{c_{s}}-4 T_{3} h_{, \sigma} h^{3}\left(1-c_{s}\right)+\frac{2 h, \sigma}{h} \frac{\dot{\sigma}}{c_{s}}+V_{, \sigma}=0
$$

To apply the ansatz (21) into Eq. (22), we then have the leading terms, which are the second term

$$
\frac{-3 H h_{I R}^{2} \sqrt{T_{3}}}{\sqrt{2 \alpha(p-1)}(-t)^{-p / 2}}
$$

and the potential term

$$
m_{n p}^{2} h_{I R}^{2} \sqrt{T_{3}} t
$$

respectively. The nonperturbative mass term is given by Eq. (9), as introduced in Section 2. The others are suppressed by the slow-varying factor $h, \sigma$ and $\frac{1}{H t}$ (where $|H t| \gg 1$ is required by inflation). By matching the leading terms, we get the results $p=2$ and $\alpha=\frac{9 H^{2}}{2 m_{n p}^{4}}$. So we obtain the solution

$$
\sigma=-h_{I R}^{2} \sqrt{T_{3}} t\left(1+\frac{9 H^{2}}{2 m_{n p}^{4} t^{2}}+\cdots\right)
$$

for a relativistic curvaton brane rotating in a warped throat.

Notice that the value range of the curvaton is constrained by the radius of the 3 -circle at the tip. In the usual case, it restrict the variation of the coordinate along the angular direction $\Delta y$ to be less than $O(1)$. Consequently, to multiply the hubble parameter on the solution (25), the angular restriction brings a constraint on the efolding number of inflation as follows,

$$
\mathcal{N}_{\mathrm{inf}} \equiv \int H d t \lesssim H \frac{\left(g_{s} M \alpha^{\prime}\right)^{\frac{1}{2}}}{h_{I R}}
$$

In addition, the solution (25) gives a rolling-down behavior for the curvaton field, which in the stringy frame shows that the rotation angle of the curvaton brane becomes more and more parallel to the 3-cycle at the tip, and the angular-dependence will finally vanish. However, the quantum fluctuation of the curvaton during inflation may be in conflict with its classical variation so that hold the curvaton on the plateau of its potential longer. Therefore, with the help of quantum fluctuation there would be more angular modes preserved, but the model may suffer from the potential tension of large backreaction. 


\section{PRIMORDIAL PERTURBATIONS OF THE CURVATON BRANE}

In the above section we have studied the background evolution of the curvaton brane during inflation. If our model indeed makes sense to the physics of the early universe, it is necessary to study the primordial perturbations generated by the curvaton field. This provides a potential discriminant between numerous curvaton models in the literature.

\section{A. Quantum fluctuations during inflation}

To begin with, we split the curvaton field $\sigma$ into $\sigma(\mathbf{x}, t)=\sigma_{0}(t)+\delta \sigma(\mathbf{x}, t)$, where $\sigma_{0}$ stands for the background field which is homogeneous and isotropic, and $\delta \sigma$ stands for the linear fluctuation which is caused by quantum effects.

Following the formulae of Ref. [24], we study the dynamics of quantum fluctuations in the spatially flat gauge, and assume no couplings to the background inflaton. In this case, we have the equation of motion describing the canonical perturbation variable in Fourier space as follows,

$$
v_{k}^{\prime \prime}+\left(c_{s}^{2} k^{2}-\frac{z^{\prime \prime}}{z}\right) v_{k} \simeq 0,
$$

where the prime denotes the derivative with respect to the conformal time $\tau \equiv \int \frac{d t}{a}$. The canonical perturbation variable is defined as

$$
v_{k} \equiv a c_{s}^{-3 / 2} \delta \sigma_{k},
$$

and a background dependent function is introduced,

$$
z \equiv a c_{s}^{-3 / 2} H^{-1} \dot{\sigma},
$$

in the above perturbation equation.

One may notice that Eq (27) has an asymptotic solution when we neglect the last term $\frac{z^{\prime \prime}}{z}$, which implies $\left|c_{s} k \tau\right| \gg 1$, and it oscillates strongly like a sine function. This feature coincides with the slow roll condition defined above, which corresponds to the case in which the effective physical wavelength is deep inside the so-call sound horizon $\frac{c_{s}}{H}$. Therefore, the modes can be regarded as states in the adiabatic Minkowski vacuum during the sub-Hubble regime. Correspondingly, we can impose a suitable initial condition

$$
v_{k}^{i n i}=\frac{e^{-i c_{s} k \tau}}{\sqrt{2 c_{s} k}},
$$

which corresponds to the Bunch-Davies vacuum.

In the inflationary background, we have the following useful approximate relation,

$$
\frac{z^{\prime \prime}}{z} \simeq \frac{2}{\tau^{2}}
$$

which implies that the power spectrum of the field fluctuations takes the form

$$
\delta \sigma=\sqrt{P_{\delta \sigma}} \simeq \frac{H_{*}}{2 \pi},
$$

after they exit the sound horizon. The subscript ' $*$ ' stands for the sound horizon crossing time for the field fluctuation.

Since during inflation the Hubble parameter is nearly constant and the field fluctuations are almost conserved after sound horizon crossing, we are able to calculate the spectral tilt of the primordial perturbations at the horizon crossing moment. This is given by

$$
n_{\sigma}-1 \equiv \frac{d \ln P_{\sigma}}{d \ln k}=-2 \epsilon_{*}+3 s_{*},
$$

where we have defined a series of slow-variation parameters,

$$
\begin{gathered}
\epsilon \equiv-\frac{\dot{H}}{H^{2}}, \\
s \equiv \frac{\dot{c}_{s}}{H c_{s}} .
\end{gathered}
$$

Note that we have ignored the square and the higher derivatives of the slow roll parameters in the above expressions. The perturbation equation is derived under the assumption that the coupling between the adiabatic and isocurvature modes is negligible. A more detailed analysis of a similar scenario where an inflation model is based on multiple branes has been given in Ref. [24]. Thus we can naturally understand the difference in the results obtained in the current model and those obtained in 7] where multiple moduli degrees of freedom are involved in a single brane. Besides, to compare the r.h.s. of Eq. (33) with that in the usual curvaton model, we can see that the correction to the spectral index from the mass term disappears in our model, because it is strongly suppressed by a term proportional to $c_{s}^{3}$.

\section{B. Curvature perturbations}

To proceed, we calculate the curvature perturbations generated by the curvaton field after inflation has ceased and the inflaton field has already decayed to radiation. During this period, the energy density of the universe is composed of $\rho_{r}$ which denotes the radiation energy density and $\rho_{\sigma}$ which is contributed by the curvaton. In this stage, the curvature perturbations are generated from the isocurvature modes since the pressure perturbations are non-adiabatic. This process ends when the perturbations become adiabatic again, which corresponds to the epoch of curvaton domination, or that of curvaton decay. The final curvature perturbations can be calculated at the moment $H=\Gamma$ based on the assumption of perturbatively instantaneous reheating of the curvaton. In 
that case we can simply consider the component curvature perturbations $\zeta_{\sigma}$ and $\zeta_{\gamma}$ on slices of uniform curvaton density and radiation density separately. According to the assumptions of the curvaton mechanism, we can neglect the perturbations from radiation and thus have the curvature perturbation expressed as,

$$
\zeta=\frac{3\left(1+w_{\sigma} \rho_{\sigma}\right)}{4 \rho_{\gamma}+3\left(1+w_{\sigma}\right) \rho_{\sigma}} \zeta_{\sigma},
$$

where $w_{\sigma} \equiv p_{\sigma} / \rho_{\sigma}$ is defined as the equation-of-state of the curvaton, and $\zeta_{\sigma}$ is given by

$$
\zeta_{\sigma}=\frac{\delta \rho_{\sigma}}{3\left(1+w_{\sigma}\right) \rho_{\sigma}} .
$$

As emphasized in Section II, the nonperturbative effect which stabilizes the Kähler moduli in the case of the Kuperstein embedding yields a quadratic potential for the curvaton brane as given in Eq. (16). Therefore, after inflation the curvaton field arrives at its vacuum state and starts to oscillate when $H=m_{n p}$. At that moment, the sound speed $c_{s}$ goes to 1 , and the energy density of the curvaton field redshifts proportional to $a^{-3}$. So we have an approximate equation-of-state $w_{\sigma}=0$ for the curvaton. In this case the energy density of the curvaton is dominated by its potential, which in the oscillating epoch roughly takes the form

$$
\rho_{\sigma} \simeq V(\sigma)=2 T_{3} h_{I R}^{4}+\frac{1}{2} m_{n p}^{2} \sigma^{2},
$$

where the first term is secondary in the oscillating epoch as will be explained in the next section.

One can obtain the linear perturbation of the curvaton energy density in the oscillating epoch as follows,

$$
\delta \rho_{\sigma_{\mathrm{osc}}}=m_{n p}^{2} \sigma_{\mathrm{osc}} \delta \sigma_{\mathrm{osc}},
$$

where $\sigma_{\text {osc }}$ is the amplitude at the beginning of its sinusoidal oscillations. It can be viewed as a function of the curvaton field $\sigma_{*}$ at Hubble exit, which is the only relevant quantity since the perturbations from the radiation fluid is supposed to be negligible. The curvature perturbation is calculated at the moment of curvaton decay, so it is convenient to define a parameter

$$
r_{\sigma} \equiv \frac{3 \rho_{\sigma \mathrm{dec}}}{4 \rho_{\gamma_{\mathrm{dec}}}+3 \rho_{\sigma \mathrm{dec}}},
$$

which characterizes the fraction of curvaton component when it starts to decay. Consequently, at fixed $\rho_{\text {osc }}$ and $\rho_{\text {dec }}$, we obtain the curvature perturbation at linear order as follows,

$$
\zeta_{g} \simeq \frac{r_{\sigma} H_{*}}{3 \pi \sigma_{\mathrm{osc}}},
$$

where we have applied the result presented in Eq. (32) as well as the approximate relation $\sigma_{\text {osc }, \sigma_{*}} \simeq 1$ when the oscillation starts with $H=m_{n p}$.

\section{Non-Gaussianities}

In the above section we have studied the primordial perturbations at linear order. It is necessary to extend the theoretical framework of this model beyond the leading order. Particularly, the investigation of non-Gaussianities may provide a powerful discriminator among numerous models describing the early universe. For example, non-Gaussianities in a single canonical field inflation model were calculated in pioneering works [43, 44] and found to be very small. Later, studies of DBI inflation models 45] predicted that a large non-Gaussianity of equilateral type can be realized in inflationary cosmology [46]. Recent developments in cosmological perturbation theory shows that a sizable nonGaussianity of local type can be obtained in bounce cosmology but that it takes on negative values [47, 48].

\section{Local type}

To expand the perturbations to second order, we are able to investigate the features of non-Gaussianities in the curvaton brane scenario. At first glance, we consider the local form. The relative magnitude of the nonlinear perturbation is conventionally specified by a parameter $f_{\mathrm{NL}}$, which is defined by

$$
f_{\mathrm{NL}} \equiv \frac{5}{3} \frac{\zeta-\zeta_{g}}{\zeta_{g}^{2}} .
$$

In this case, it is convenient to use the $\delta \mathcal{N}$ formalism to study the non-Gaussian perturbations by virtue of the separate universe assumption [49, 50, 51]. Starting from an initial flat slice at time $t_{\mathrm{osc}}$, the curvature perturbation can be generally expanded as

$$
\zeta(t, \vec{x})=\mathcal{N}_{, \sigma} \delta \sigma+\frac{1}{2} \mathcal{N}_{, \sigma \sigma} \delta \sigma^{2}+\ldots,
$$

where $\mathcal{N}(t, \vec{x}) \equiv \ln \left[a_{\mathrm{dec}} / a_{\text {osc }}\right]$ is the amount of expansion to the final slice of uniform energy density at curvaton decay. To compare the first term in the r.h.s. of Eq. (43) with the linear curvature perturbation obtained in Eq. (41), we can read $\mathcal{N}_{, \sigma_{*}}=2 r_{\sigma} / 3 \sigma_{\text {osc }}$. Differentiating this term with respect to $\sigma_{*}$ again and applying the definition of $f_{\mathrm{NL}}$, we can obtain

$$
f_{\mathrm{NL}}=\frac{5}{6} \frac{\mathcal{N}_{, \sigma_{*} \sigma_{*}}}{\mathcal{N}_{, \sigma_{*}}^{2}} \simeq-\frac{5}{3}-\frac{5 r_{\sigma}}{6}+\frac{5}{4 r_{\sigma}} .
$$

This form precisely agrees with the result obtained in a normal curvaton model [5], and more detailed studies of this parameter were present in [50, 52, 53].

\section{Equilateral type}

The above result shows that the local non-Gaussianity in our model nicely coincides with that given in a general 
curvaton model. For this case, we are unable to differentiate the model of curvaton brane from the standard curvaton model by observing the local non-Gaussianity. So we need to study more details of the nonlinear perturbations generated in our model. In particular, we are interested in the non-Gaussianity of equilateral type.

The presence of interactions in the perturbation Lagrangian leads to non-Gaussianities. To start, we define the power spectrum $P_{\zeta}$ and bispectrum $B_{\zeta}$ as follows,

$$
\begin{aligned}
\left\langle\zeta_{k_{1}} \zeta_{k_{2}}\right\rangle= & (2 \pi)^{3} \delta^{3}\left(\vec{k}_{1}+\vec{k}_{2}\right) P_{\zeta}\left(k_{1}\right) \\
\left\langle\zeta_{k_{1}} \zeta_{k_{2}} \zeta_{k_{3}}\right\rangle= & (2 \pi)^{3} \delta^{3}\left(\vec{k}_{1}+\vec{k}_{2}+\vec{k}_{3}\right) \\
& \times B_{\zeta}\left(k_{1}, k_{2}, k_{3}\right)
\end{aligned}
$$

and then these two spectra can be related in terms of a $k$-dependent nonlinearity parameter $f_{N L}$,

$$
B_{\zeta}\left(k_{1}, k_{2}, k_{3}\right)=\frac{3}{10}(2 \pi)^{4} \frac{\sum k_{i}^{3}}{\prod k_{i}^{3}} P_{\zeta}^{2} f_{N L}\left(k_{1}, k_{2}, k_{3}\right),
$$

in momentum space.

It is easiest to work in the interaction picture, in which the three-point correlator to leading order is given by

$$
\begin{aligned}
\left.\left\langle\zeta_{k_{1}} \zeta_{k_{2}} \zeta_{k_{3}}\right\rangle\right|_{\mathrm{dec}} & =i \int_{t_{i}}^{t_{\mathrm{dec}}} d t^{\prime}\left\langle\left[\zeta_{k_{1}} \zeta_{k_{2}} \zeta_{k_{3}}, L_{3}\left(t^{\prime}\right)\right]\right\rangle \\
& =i \mathcal{N}_{, \sigma_{*}}^{3} \int_{t_{i}}^{t_{*}} d t^{\prime}\left\langle\left[\delta \sigma_{k_{1}} \delta \sigma_{k_{2}} \delta \sigma_{k_{3}}, L_{3}\left(t^{\prime}\right)\right]\right\rangle
\end{aligned}
$$

where the $\delta \mathcal{N}$ formalism was applied. For a generic curvaton model the integral stops at the moment of curvaton decay. Here $t_{i}$ corresponds to the initial time before which there are any non-Gaussianities. The square parentheses indicate the commutator, and $L_{3}$ is the interaction Lagrangian which will be performed in the following. In the second expression of Eq. (48) we have neglected the integral from the Hubble exit moment to the curvaton decay, due to the assumption of a curvaton model that the number of efolds from the end of inflation to the beginning of the oscillations is completely unperturbed.

If we have computed the three point correlators of curvaton field fluctuations, the nonlinearity parameter can be obtained by making use of the above equations. With an assumption of weak coupling between the fields, we perturb the sound speeds in the quadratic lagrangian and then obtain the lagrangian with the leading order terms up to cubic parts,

$$
\mathcal{L}_{3} \supseteq \frac{a^{3}}{2 c_{s}{ }^{5} \dot{\sigma}}\left[\delta \dot{\sigma}^{3}-\frac{c_{s}{ }^{2}}{a^{2}} \delta \dot{\sigma}(\nabla \delta \sigma)^{2}\right] .
$$

Correspondingly, the dominant terms in the interaction Hamiltonian in Fourier space are given by

$$
H_{\text {int }} \supseteq \int d k^{3}\left[-\frac{a^{3}}{2 c_{s}{ }^{5} \dot{\sigma}}\left(\delta \dot{\sigma}^{3}+\frac{c_{s}^{2}}{a^{2}} k^{2} \delta \dot{\sigma} \delta \sigma^{2}\right)\right] .
$$

Then we decompose the field fluctuations in canonical quantization process,

$$
\begin{aligned}
\delta \sigma_{k}(t) & =u(\vec{k}) a_{\vec{k}}+u^{*}(-\vec{k}) a_{-\vec{k}}^{\dagger}, \\
u(\vec{k}) & =\frac{H}{\sqrt{2 k^{3}}}\left(1+i c_{s} k \tau\right) e^{-i c_{s} k \tau},
\end{aligned}
$$

with the creation and annihilation operators defined by $\left[a_{\vec{k}}, a_{\vec{k}^{\prime}}^{\dagger}\right]=\delta\left(\vec{k}-\vec{k}^{\prime}\right)$.

Since we have obtained the interaction Hamiltonian and the modes of the field fluctuations, we are now able to calculate the three point correlator of equilateral form,

$$
\begin{aligned}
\left\langle\delta \sigma_{k}^{3}\right\rangle & =-i \int d t\left\langle\left[\delta \sigma_{k}^{3}, H_{i n t}\right]\right\rangle \\
& \sim-\frac{H_{*}^{5}}{c_{s}^{2} \dot{\sigma}_{*} k^{6}}(2 \pi)^{3} \delta\left(\sum \vec{k}_{i}\right) .
\end{aligned}
$$

From Eqs. (50) and the above result, we can see that $H_{\text {int }} \sim 1 / c_{s}{ }^{2}$. This roughly agrees with the result in usual single DBI inflation which is proportional to $1 / c_{s}^{2}[46$. To be explicit, we combine Eqs. (41), (47) and (48) and eventually have the non-Gaussianity parameter of equilateral type,

$$
f_{\mathrm{NL}} \simeq-\frac{\sigma_{o s c} H_{*}}{2 r_{\sigma} c_{s}^{2} h_{I R}^{2} \sqrt{T_{3}}} .
$$

From the above analysis, we learn that the nonGaussianity of equilateral type in the model of curvaton brane can be amplified both by small $r_{\sigma}$ and $c_{s}^{2}$. We may understand this phenomenon as follows. The amplification of non-Gaussianities from $r_{\sigma}$ is inherited from the physical feature of a general curvaton model. Moreover, when those perturbation modes exit the Hubble radius during inflation, the sound speed is also small due to the relativistic motion of the curvaton brane in the warped throat, and thus leads to significant equilateral non-Gaussianity signals.

Note that in the above computations we assumed that the curvaton enters the oscillating epoch very soon after inflation. So an approximate relation $\sigma_{\mathrm{osc}, \sigma_{*}} \simeq 1$ can be used to obtain an estimate. Under this estimate there is

$$
\sigma_{o s c} \simeq 5 h_{I R}^{2} \sqrt{T_{3}} / m_{n p}
$$

and so $f_{\mathrm{NL}} \simeq-5 H_{*} / 2 r_{\sigma} c_{s}^{2} m_{n p}$. It is worth extending the above analysis to more general cases.

\section{CONSISTENCY RELATIONSHIP AND CONSTRAINTS}

In this section, we shall discuss the consistency conditions and observational constraints on the phase space of the parameters appearing in our model.

To our current knowledge, the most powerful observational constraints on models of early universe are provided by the $\mathrm{CMB}$ experiments. In order to link the 
observations with the theoretical studies, let us make a short summary of the observable predictions obtained in our model. In our model, the amplitudes of the scalar and tensor perturbation spectra are given by

$$
P_{\zeta}=\frac{r_{\sigma}^{2} m_{n p}^{2} H_{*}^{2}}{225 \pi^{2} h_{I R}^{4} T_{3}}, \quad P_{T}=\frac{2 H_{*}^{2}}{\pi^{2} m_{p l}^{2}},
$$

respectively, where $m_{p l}=1 / \sqrt{8 \pi G}$. The corresponding spectral indices are given by

$$
n_{\zeta}=1-2 \epsilon_{*}+3 s_{*}, \quad n_{t}=-2 \epsilon_{*},
$$

and the spectral tilt of the scalar perturbation spectrum is the same as that of the iso-curvature modes during inflation as shown in Eq. (33). The tensor-to-scalar ratio takes the form,

$$
r_{T} \equiv \frac{P_{T}}{P_{\zeta}}=\frac{450 h_{I R}^{4} T_{3}}{r_{\sigma}^{2} m_{n p}^{2} m_{p l}^{2}} .
$$

Going beyond the Gaussian statistics in the scalar perturbation spectrum, the non-Gaussian fluctuations are characterized in terms of the nonlinearity parameter $f_{\mathrm{NL}}$. In the local and equilateral limits, the leading order contributions to this parameter are expressed as,

$$
f_{\mathrm{NL}}^{\text {local }} \simeq-\frac{5}{3}-\frac{5 r_{\sigma}}{6}+\frac{5}{4 r_{\sigma}}, \quad f_{\mathrm{NL}}^{\text {equil }} \simeq-\frac{5 H_{*}}{2 r_{\sigma} c_{s *}^{2} m_{n p}},
$$

respectively.

Since in our model the position of the curvaton brane is fixed at the tip of the warped throat, it suffers from very few theoretical constraints compared to usual brane inflation models, namely, the bounds from the throat 54 and bulk volume 55] and the primordial tensor modes [56] can be circumvented delicately. However, one still needs to be careful of the local gravitational backreaction of the probe brane on the throat. In the usual case this effect is negligible provided $4 \pi g_{s} \alpha^{\prime 2} \ll c_{s}^{2} L^{4}$ [14], and so gives a bound on the sound speed of the curvaton brane $c_{s_{*}}^{2} \gg 0.6 / M K$ during inflation.

Combining the Wilkinson Microwave Anisotropy Probe five year (WMAP5) data [57] with the distance measurements from the Type Ia supernovae (SN) [58] and the Baryon Acoustic Oscillations (BAO) in the distribution of galaxies [59], the parameters of primordial cosmological perturbations have been accurately determined by a group of stringent limits as follows: $P_{\zeta}=$ $(2.445 \pm 0.096) \times 10^{-9}, n_{\zeta}=0.96 \pm 0.013$ at the pivot scale $k=0.002 \mathrm{Mpc}^{-1}, r_{T}<0.22$ at the $2 \sigma$ level with a prior assumption of no running of the spectral index, and further $-9<f_{\mathrm{NL}}^{\text {local }}<111$ and $-151<f_{\mathrm{NL}}^{\text {equil }}<253$ at the $2 \sigma$ level for the local and equilateral limits, respectively.

Moreover, the number of efoldings of inflation is also constrained by cosmological observations. However, the reheating process after the primordial epoch is quite model-dependent and critically dependent on the reheating temperature. Up to now, the allowed reheating temperature can be anywhere between $1 \mathrm{MeV}$ and $10^{16} \mathrm{GeV}$ in principle. So it makes the measurement of the inflationary efolding number undetermined. In the current work, we take the simplest example of instantaneous curvaton reheating where the curvaton decays to standard-model particles momentarily once $H=\Gamma$. Therefore, we simply assume the inflationary efolding number takes the value $\mathcal{N}_{\text {inf }}=50$, which is a quite reasonable value allowed by observations. We would like to leave the more detailed study of the reheating process of the curvaton brane model to future studies.

Additionally, there is one extra theoretical constraints from the requirement that the scalar perturbations generated by the inflaton field should be negligible during the period of curvaton oscillations. We assume that the inflaton field satisfies the traditional slow-roll conditions, and hence the curvature perturbation during inflation are given by the power spectrum $P_{S}=\frac{H_{*}^{2}}{8 \pi^{2} m_{p l}^{2} \epsilon_{*}}$. Similar to the definition of the tensor-to-scalar ratio, one can define a new parameter

$$
r_{S} \equiv \frac{P_{S}}{P_{\zeta}}=\frac{225 h_{I R}^{4} T_{3}}{8 r_{\sigma}^{2} m_{n p}^{2} m_{p l}^{2} \epsilon_{*}},
$$

which has to satisfy the condition $r_{S} \ll 1$.

Taking into account all the conditions introduced above, we find the available stringy parameters most stringently constrained by observations are $g_{s}$ and $M$. In Fig. 2, 3] and 4, we present our numerical calculations of the permitted regime for $g_{s}$ and $M$ by fixing the total charge number of the background $M K$. Our results imply that the allowed phase space of the $g_{s}-M$ plane with a large $M K$ is much bigger than that with a small one. This feature is similar to that of a traditional DBI inflation model, where a large value of $M K$ can provide a small enough warp factor; however, the limits on stringy parameters are much weaker in our case. This difference is mainly due to the particular feature of our model that the curvaton position keeps still during the epoch of inflation.

In addition to the constraints on the stringy parameters performed above, we can also obtain the constraint on the slow-roll parameter $\epsilon_{*}$ as $0.0174<\epsilon_{*}<0.0226$. Moreover, in order to make sure that the equilateral nonGaussianity generated in our model is consistent with the WMAP5 data, the fraction of curvaton density $r_{\sigma}$ in the oscillating epoch needs to be of order $O(1)$. This is selfconsistent with the previous assumption that the universe is dominated by the curvaton field after inflation.

To conclude, in this section we have confronted our model with the latest cosmological observations. Under the priors of the inflationary efolding number and the background charge number, we derived that there is a sizable allowed region for the phase space of this model. It illustrates that cosmological observations can be nicely satisfied in our model. However, it is crucial to perform a global analysis which combines both the stringy parameters appearing in the model and the standard cosmological parameters without any artificial assumptions. We 


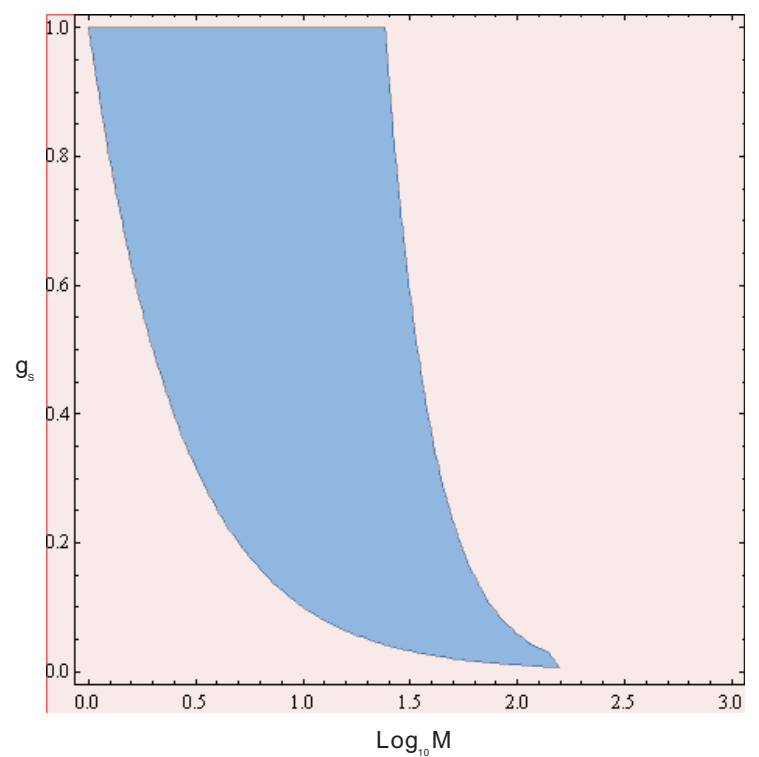

FIG. 2: Two dimensional constraints on $g_{s}$ and $M$ from current observations, assuming a curvaton-dominated universe after inflation. The blue region is allowed by current observations. In the numerical computation, $M K=10^{3}$ and $\mathcal{N}=50$ are chosen as priors.

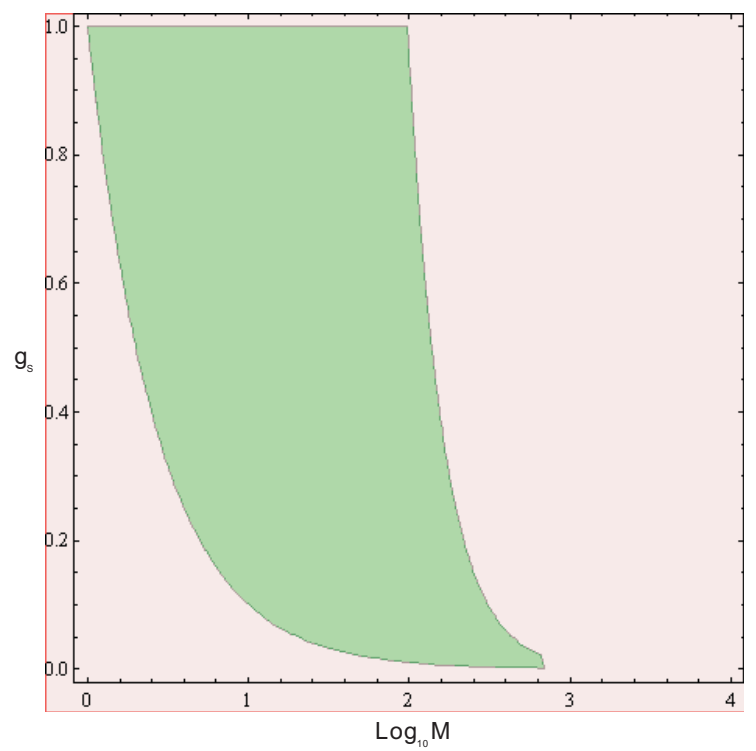

FIG. 3: Two dimensional constraints on $g_{s}$ and $M$ from current observations, assuming a curvaton-dominated universe after inflation. The green region is allowed by current observations. In the numerical computation, $M K=10^{4}$ and $\mathcal{N}=50$ are chosen as priors.

will leave this work to a future study.

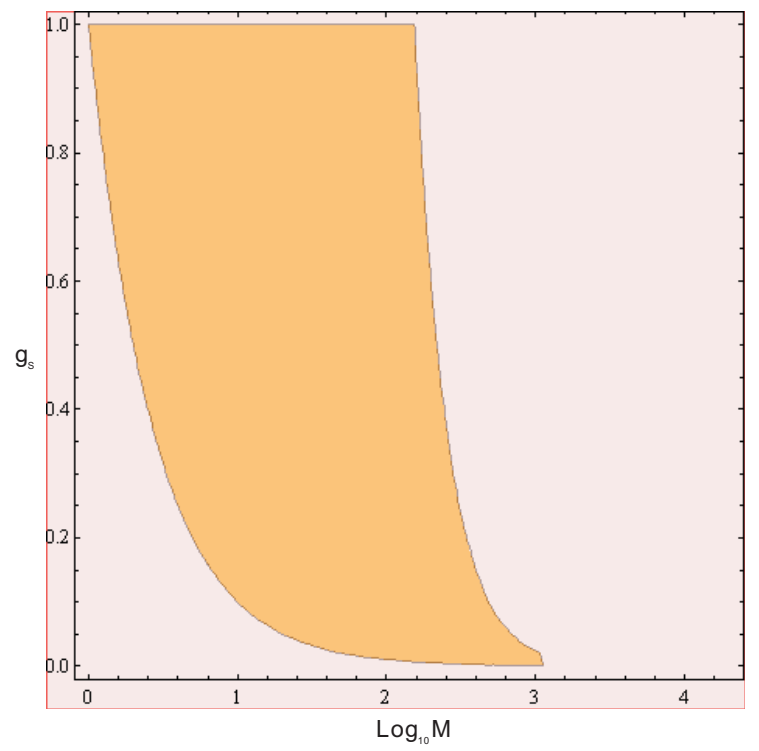

FIG. 4: Two dimensional constraints on $g_{s}$ and $M$ from current observations, assuming a curvaton-dominated universe after inflation. The orange region is allowed by current observations. In the numerical computation, $M K=2 \times 10^{4}$ and $\mathcal{N}=50$ are chosen as priors.

\section{DISCUSSION AND CONCLUSIONS}

In this paper, we have presented a curvaton model obtained by considering a $\bar{D}$-brane in a KS throat with approximate isometries from string theory. In this model, a $\overline{D 3}$-brane is stretched out along the $4 \mathrm{D}$ external space, and can be localized at the tip of the KS throat because of the gauge forces. Adding the nonperturbative effect of the Kähler moduli stabilization, its angular coordinates can play a role of the curvaton while the other degrees of freedom (for example, the radial motion of the $\overline{D 3}$-brane) are almost frozen. This scenario can be embedded into the framework of inflation models in terms of multiple branes, in which the $\overline{D 3}$-brane rotates relativistically.

Based on the formulae developed in Ref. [23] and [24], we have studied the background evolution and the primordial perturbations of this curvaton model. The solution of the background dynamics of the curvaton is consistent with the assumptions of the nonperturbative potential of moduli stabilization, and so ensures the feasibility of our model. The process of converting the primordial fluctuations of the curvaton into curvature perturbations takes place after inflation, and is similar to what happens in the usual curvaton mechanism. We would like to emphasize that the final curvature perturbations are calculated under the assumption that the curvaton decays immediately after the oscillation period ceases. Such a fast decay rate is also suggested by the stringy reheating configuration, in which one needs to turn on certain SUSY-breaking deformations of the KS background 60 in order to avoid redundant long-lived Kaluza-Klein (KK) modes [61, 62] and have a correct relic 
density for DM candidates [60, 63]. However, the precise duration of the decay process is not addressed in the current work. This would alter the efficiency of the conversion of curvature perturbations and the precise determination of the inflationary efolding number.

Our model is related to many other interesting issues which deserve intensive studies in the future. For example, as mentioned below Eq. (12), this model is expected to possess nontrivial kinetic couplings at fourth order from the geometric brane deformation. Therefore it may leave important signals on the trispectra which would render the model distinguishable from other multi-field inflation models with non-standard kinetic terms [64, 65, 66, 67, 68, 69, 70, 71]. In addition, a lesson from string theory suggests that a more reasonable expression of the multi-brane dynamics ought to be described by a matrix in the framework of nonabelian background [72], which shows a potential connection between the multi-brane scenario and the matrix inflation model investigated recently [73, 74].

\section{Acknowledgements}

We would like to thank Aaron Berndsen, Robert Brandenberger and Xingang Chen for helpful discussions and comments on our work. Y.F.C. acknowledges Prof. Xinmin Zhang for extensive support of his research. Y.F.C. also thanks the Canadian Institute for Theoretical Astrophysics, McGill University, Perimeter Institute, Simon Fraser University, and the University of British Columbia for hospitality. The research of J.Z. and Y.S.P is supported in part by NSFC under Grant No:10775180, in part by the Scientific Research Fund of GUCAS (NO.055101BM03), in part by CAS under Grant No: KJCX3-SYW-N2.
[1] D. H. Lyth and D. Wands, Phys. Lett. B 524, 5 (2002) arXiv:hep-ph/0110002.

[2] S. Mollerach, Phys. Rev. D 42, 313 (1990).

[3] A. Linde and V. Mukhanov, Phys. Rev. D 56, 535 (1997) arXiv:astro-ph/9610219.

[4] K. Enqvist and M. S. Sloth, Nucl. Phys. B 626, 395 (2002) arXiv:hep-ph/0109214.

[5] D. H. Lyth, C. Ungarelli and D. Wands, Phys. Rev. D 67, 023503 (2003) arXiv:astro-ph/0208055.

[6] D. H. Lyth and D. Wands, Phys. Rev. D 68, 103516 (2003) arXiv:astro-ph/0306500.

[7] S. Li, Y. F. Cai and Y. S. Piao, Phys. Lett. B 671, 423 (2009) arXiv:0806.2363 [hep-ph]].

[8] G. R. Dvali and S. H. H. Tye, Phys. Lett. B 450, 72 (1999) arXiv:hep-ph/9812483.

[9] S. Kachru, R. Kallosh, A. Linde, J. M. Maldacena, L. P. McAllister and S. P. Trivedi, JCAP 0310, 013 (2003) arXiv:hep-th/0308055.

[10] E. Silverstein and D. Tong, Phys. Rev. D 70, 103505 (2004) arXiv:hep-th/0310221.

[11] X. Chen, Phys. Rev. D 71, $063506 \quad$ (2005) arXiv:hep-th/0408084.

[12] M. X. Huang, G. Shiu and B. Underwood, Phys. Rev. D 77, 023511 (2008) arXiv:0709.3299 [hep-th]].

[13] X. Chen, JHEP 0508, 045 arXiv:hep-th/0501184.

[14] D. A. Easson, R. Gregory, D. F. Mota, G. Tasinato and I. Zavala, JCAP 0802, 010 (2008) arXiv:0709.2666 [hepth]].

[15] X. Chen, JCAP 0812, 009 (2008) arXiv:0807.3191 [hepth]].

[16] R. Bean, S. E. Shandera, S. H. Henry Tye and J. Xu, JCAP 0705, 004 (2007) arXiv:hep-th/0702107.

[17] H. V. Peiris, D. Baumann, B. Friedman and A. Cooray, Phys. Rev. D 76, 103517 (2007) arXiv:0706.1240 [astro$\mathrm{ph}]$.

[18] R. Bean, X. Chen, H. Peiris and J. Xu, Phys. Rev. D 77, 023527 (2008) arXiv:0710.1812 [hep-th]].

[19] D. Baumann, A. Dymarsky, I. R. Klebanov, L. McAllister and P. J. Steinhardt, Phys. Rev. Lett. 99, 141601 (2007) arXiv:0705.3837 [hep-th]].

[20] A. Krause and E. Pajer, JCAP 0807, 023 (2008) arXiv:0705.4682 [hep-th]].

[21] D. Baumann, A. Dymarsky, I. R. Klebanov and L. McAllister, JCAP 0801, 024 (2008) arXiv:0706.0360 [hep-th]].

[22] O. DeWolfe, L. McAllister, G. Shiu and B. Underwood, JHEP 0709, 121 (2007) arXiv:hep-th/0703088.

[23] Y. F. Cai and W. Xue, Phys. Lett. B 680, 395 (2009) arXiv:0809.4134 [hep-th]].

[24] Y. F. Cai and H. Y. Xia, Phys. Lett. B 677, 226 (2009) arXiv:0904.0062 [hep-th]].

[25] I. R. Klebanov and M. J. Strassler, JHEP 0008, 052 (2000) arXiv:hep-th/0007191.

[26] S. B. Giddings, S. Kachru and J. Polchinski, Phys. Rev. D 66, 106006 (2002) arXiv:hep-th/0105097.

[27] S. Kachru, R. Kallosh, A. Linde and S. P. Trivedi, Phys. Rev. D 68, 046005 (2003) arXiv:hep-th/0301240.

[28] See also an earlier different implement with tachyon fields: Y. S. Piao, R. G. Cai, X. M. Zhang and Y. Z. Zhang, Phys. Rev. D 66, 121301 (2002) arXiv:hep-ph/0207143.

[29] O. Aharony, S. S. Gubser, J. M. Maldacena, H. Ooguri and Y. Oz, Phys. Rept. 323, 183 (2000) arXiv:hep-th/9905111.

[30] R. C. Myers, JHEP 9912, 022 (1999) arXiv:hep-th/9910053.

[31] A. Ceresole, G. Dall'Agata, R. D'Auria and S. Ferrara, Phys. Rev. D 61, 066001 (2000) arXiv:hep-th/9905226.

[32] O. DeWolfe, S. Kachru and H. L. Verlinde, JHEP 0405, 017 (2004) arXiv:hep-th/0403123.

[33] O. Aharony, Y. E. Antebi and M. Berkooz, Phys. Rev. D 72, 106009 (2005) arXiv:hep-th/0508080.

[34] M. Berg, M. Haack and B. Kors, Phys. Rev. D 71, 026005 (2005) arXiv:hep-th/0404087.

[35] D. Baumann, A. Dymarsky, I. R. Klebanov, J. M. Maldacena, L. P. McAllister and A. Murugan, JHEP 0611, 031 (2006) arXiv:hep-th/0607050.

[36] S. Kuperstein, JHEP 0503, $014 \quad$ (2005) 
arXiv:hep-th/0411097.

[37] A. Klemm, B. Lian, S. S. Roan and S. T. Yau, Nucl. Phys. B 518, 515 (1998) arXiv:hep-th/9701023.

[38] C. Herdeiro, S. Hirano and R. Kallosh, JHEP 0112, 027 (2001) arXiv:hep-th/0110271.

[39] K. Dasgupta, C. Herdeiro, S. Hirano and R. Kallosh, Phys. Rev. D 65, 126002 (2002) arXiv:hep-th/0203019].

[40] C. Germani, N. E. Grandi and A. Kehagias, Class. Quant. Grav. 25, 135004 (2008) arXiv:hep-th/0611246.

[41] C. Germani and M. Liguori, Gen. Rel. Grav. 41, 191 (2009) arXiv:0706.0025 [astro-ph]].

[42] T. Kobayashi and S. Mukohyama, JCAP 0907, 032 (2009) arXiv:0905.2835 [hep-th]].

[43] V. Acquaviva, N. Bartolo, S. Matarrese and A. Riotto, Nucl. Phys. B 667, 119 (2003) arXiv:astro-ph/0209156.

[44] J. M. Maldacena, JHEP 0305, 013 (2003) arXiv:astro-ph/0210603.

[45] M. Alishahiha, E. Silverstein and D. Tong, Phys. Rev. D 70, 123505 (2004) arXiv:hep-th/0404084.

[46] X. Chen, M. x. Huang, S. Kachru and G. Shiu, JCAP 0701, 002 (2007) arXiv:hep-th/0605045.

[47] Y. F. Cai, W. Xue, R. Brandenberger and X. Zhang, JCAP 0905, 011 (2009) arXiv:0903.0631 [astro-ph.CO]].

[48] Y. F. Cai, W. Xue, R. Brandenberger and X. m. Zhang, JCAP 0906, 037 (2009) arXiv:0903.4938 [hep-th]].

[49] M. Sasaki and E. D. Stewart, Prog. Theor. Phys. 95, 71 (1996) arXiv:astro-ph/9507001.

[50] D. H. Lyth and Y. Rodriguez, Phys. Rev. Lett. 95, 121302 (2005) arXiv:astro-ph/0504045.

[51] For earlier studies we refer to: A. A. Starobinsky, JETP Lett. 42 (1985) 152 [Pisma Zh. Eksp. Teor. Fiz. 42 (1985) $124]$.

[52] Q. G. Huang, Phys. Lett. B 669, 260 (2008) arXiv:0801.0467 [hep-th]].

[53] Q. G. Huang and Y. Wang, JCAP 0809, 025 (2008) arXiv:0808.1168 [hep-th]].

[54] D. Baumann and L. McAllister, Phys. Rev. D 75, 123508 (2007) arXiv:hep-th/0610285.

[55] X. Chen, S. Sarangi, S. H. Henry Tye and J. Xu, JCAP 0611, 015 (2006) arXiv:hep-th/0608082.
[56] J. E. Lidsey and I. Huston, JCAP 0707, 002 (2007) arXiv:0705.0240 [hep-th]].

[57] E. Komatsu et al. [WMAP Collaboration], Astrophys. J. Suppl. 180, 330 (2009) arXiv:0803.0547 [astro-ph]].

[58] M. Kowalski et al. [Supernova Cosmology Project Collaboration], Astrophys. J. 686, 749 (2008) arXiv:0804.4142 [astro-ph]].

[59] D. J. Eisenstein et al. [SDSS Collaboration], Astrophys. J. 633, 560 (2005) arXiv:astro-ph/0501171.

[60] A. Berndsen, J. M. Cline and H. Stoica, Phys. Rev. D 77, 123522 (2008) arXiv:0710.1299 [hep-th]].

[61] L. Kofman and P. Yi, Phys. Rev. D 72, 106001 (2005) arXiv:hep-th/0507257.

[62] J. F. Dufaux, L. Kofman and M. Peloso, Phys. Rev. D 78, 023520 (2008) arXiv:0802.2958 [hep-th]].

[63] A. R. Frey, R. J. Danos and J. M. Cline, JHEP 0911, 102 (2009) arXiv:0908.1387 [hep-th]].

[64] S. Renaux-Petel and G. Tasinato, JCAP 0901, 012 (2009) arXiv:0810.2405 [hep-th]].

[65] X. Gao and B. Hu, JCAP 0908, 012 (2009) arXiv:0903.1920 [astro-ph.CO]].

[66] S. Mizuno, F. Arroja, K. Koyama and T. Tanaka, Phys. Rev. D 80, 023530 (2009) arXiv:0905.4557 [hep-th]].

[67] C. T. Byrnes and G. Tasinato, JCAP 0908, 016 (2009) arXiv:0906.0767 [astro-ph.CO]].

[68] X. Gao, M. Li and C. Lin, JCAP 0911, 007 (2009) arXiv:0906.1345 [astro-ph.CO]].

[69] S. Mizuno, F. Arroja and K. Koyama, Phys. Rev. D 80, 083517 (2009) arXiv:0907.2439 [hep-th]].

[70] D. Battefeld and T. Battefeld, JCAP 0911, 010 (2009) arXiv:0908.4269 [hep-th]].

[71] X. Chen and Y. Wang, arXiv:0911.3380 [hep-th].

[72] W. Taylor and M. Van Raamsdonk, Nucl. Phys. B 573, 703 (2000) arXiv:hep-th/9910052.

[73] A. Ashoorioon, H. Firouzjahi and M. M. Sheikh-Jabbari, JCAP 0906, 018 (2009) arXiv:0903.1481 [hep-th]].

[74] A. Berndsen, J. E. Lidsey and J. Ward, arXiv:0908.4252 [hep-th]. 